\title{
Optimal control problems with a continuous inequality constraint on the state and the control *
}

\author{
R. C. Loxton $^{\mathrm{a}}$, K. L. Teo ${ }^{\mathrm{a}}$, V. Rehbock ${ }^{\mathrm{a}}$, K. F. C. Yiu ${ }^{\mathrm{b}}$ \\ ${ }^{a}$ Department of Mathematics and Statistics, Curtin University of Technology, Perth, Australia \\ ${ }^{\mathrm{b}}$ Department of Applied Mathematics, The Hong Kong Polytechnic University, Hong Kong
}

\begin{abstract}
We consider an optimal control problem with a nonlinear continuous inequality constraint. Both the state and the control are allowed to appear explicitly in this constraint. By discretizing the control space and applying a novel transformation, a corresponding class of semi-infinite programming problems is derived. A solution of each problem in this class furnishes a suboptimal control for the original problem. Furthermore, we show that such a solution can be computed efficiently using a penalty function method. On the basis of these two ideas, an algorithm that computes a sequence of suboptimal controls for the original problem is proposed. Our main result shows that the cost of these suboptimal controls converges to the minimum cost. For illustration, an example problem is solved.
\end{abstract}

Key words: Optimal control, Nonlinear control systems, Nonlinear programming, Constraints.

\section{Introduction}

Many dynamic control processes have requirements that must be satisfied at every point along a trajectory. Examples include container cranes (see Sakawa \& Shindo (1982)), batch crystallization processes (see Rehbock \& Livk (2007)), anti-cancer drugs (see Martin (1992)), and solar-powered vehicles (see Gates \& Westcott (1996)). Computing an optimal control law for such systems is challenging, as the trajectory requirements give rise to constraints that are imposed continuously over the entire time horizon in the corresponding optimal control problem. These types of constraints are called continuous, path, pointwise, or all-time constraints in the literature, and they differ significantly from standard interiorpoint or terminal constraints. In fact, one continuous constraint is equivalent to an uncountable number of conventional constraints.

\footnotetext{
* This paper was not presented at any IFAC meeting. The second author is supported by the National Natural Science Foundation of China under Grant 60704003 and a grant from the Australian Research Council. The last author is supported by RGC Grant PolyU. 5321/07E and the Research Committee of The Hong Kong Polytechnic University.

Email addresses: R.Loxton@curtin.edu.au (R. C. Loxton), K.L.Teo@curtin.edu.au (K. L. Teo), V.Rehbock@curtin.edu.au (V. Rehbock), macyiu@polyu.edu.hk (K. F. C. Yiu).
}

Several maximum principles have been derived for optimal control problems with continuous constraints (see Hartl, Sethi, \& Vickson (1995) for a comprehensive survey). These principles furnish the necessary conditions for optimality. The complexity and scale of applied problems, however, often prohibits the direct use of analytical results. Thus, many reliable numerical techniques for determining an approximate solution have been proposed in the literature. These include discretization methods (Büskens \& Maurer (2000), Chen \& Vassiliadis (2005), Gerdts \& Kunkel (2008)), non-smooth Newton methods (Gerdts (2008a), Gerdts (2008b)), feasible direction methods (Pytlak \& Vinter (1998), Pytlak \& Vinter (1999)), and control parametrization methods (Goh \& Teo (1988), Teo \& Jennings (1989), Teo, Goh, \& Wong (1991)).

Control parametrization, in particular, is a versatile approach that has been successfully applied to a wide variety of practical problems. It involves approximating the control function by a linear combination of basis functions, so that the original optimal control problem is approximated by a special type of nonlinear optimization problem. If the gradient of the cost function with respect to each of the decision parameters can be computed, then standard optimization methods, such as sequential quadratic programming (see Nocedal \& Wright (1999)), are applicable. For this reason, the derivation of 
appropriate gradient formulae is paramount to the successful implementation of the control parametrization approach. Note though, that since the influence of the parameters on the cost and constraint functions is not explicit, deriving such formulae is normally a non-trivial task.

The first control parametrization method capable of handling continuous constraints was introduced by Goh \& Teo (1988). This method employs a simple transformation to convert continuous constraints on the state and the control into an equivalent conventional constraint. The parametrized form of this equivalent constraint, however, does not satisfy the usual constraint qualification condition. Thus, numerical convergence cannot be guaranteed if standard optimization algorithms are applied. In fact, convergence rarely occurs in practice, although the method does give good approximate results.

To overcome this limitation, another algorithm - also based on control parametrization - was developed by Teo \& Jennings (1989). It is based on a novel approximation scheme, whereby each continuous constraint is replaced by an approximate inequality constraint. A solution of the original problem is then obtained by solving a sequence of approximate problems. Powerful convergence analysis is available to justify this approximation strategy. Nevertheless, these convergence results are only guaranteed to hold for problems with pure-state continuous constraints; constraints containing the control explicitly are not allowed. This shortcoming serves as the motivation for our current work. In this paper, we propose a new method that is capable of handling more general continuous constraints. We prove that the method has strong convergence properties. Furthermore, it can be readily implemented using existing optimization software. Thus, the greatest virtue of control parametrization - ease of implementation - is preserved.

\section{Problem formulation}

Consider the following nonlinear dynamic system:

$$
\dot{\mathbf{x}}(t)=\mathbf{f}(\mathbf{x}(t), \mathbf{u}(t)), \quad t \in[0, T],
$$

and

$$
\mathbf{x}(0)=\mathbf{x}^{0},
$$

where $T>0$ is a given terminal time; $\mathbf{x}(t) \in \mathbb{R}^{n}$ is the system state at time $t ; \mathbf{x}^{0} \in \mathbb{R}^{n}$ is a given initial state; $\mathbf{u}(t) \in \mathbb{R}^{r}$ is the control at time $t$; and $\mathbf{f}: \mathbb{R}^{n} \times \mathbb{R}^{r} \rightarrow \mathbb{R}^{n}$ is a given function. Define

$$
\Upsilon:=\left\{\gamma \in \mathbb{R}^{r}: \alpha_{i} \leq \gamma_{i} \leq \beta_{i}, i=1, \ldots, r\right\},
$$

where $\alpha_{i}$ and $\beta_{i}, i=1, \ldots, r$, are given constants. A piecewise continuous function $\mathbf{u}:[0, T] \rightarrow \mathbb{R}^{r}$ such that $\mathbf{u}(t) \in \Upsilon$ for almost all $t \in[0, T]$ is called an admissible control for the dynamic system (2.1)-(2.2). Let $\mathcal{U}$ denote the class of all such admissible controls. We assume that the following two conditions are satisfied.

Assumption 2.1. The function $\mathbf{f}$ is continuously differentiable.

Assumption 2.2. There exists a real number $L_{1}>0$ such that

$$
\|\mathbf{f}(\mathbf{x}, \mathbf{u})\| \leq L_{1}(1+\|\mathbf{x}\|), \quad(\mathbf{x}, \mathbf{u}) \in \mathbb{R}^{n} \times \Upsilon
$$

Hence, for each $\mathbf{u} \in \mathcal{U}$, there exists a unique absolutely continuous function $\mathbf{x}(\cdot \mid \mathbf{u})$ satisfying the dynamics $(2.1)$ almost everywhere and the initial condition (2.2) (see Theorem 3.3.3 of Ahmed (2006)).

Consider the following continuous inequality constraint:

$$
h(\mathbf{x}(t \mid \mathbf{u}), \mathbf{u}(t)) \geq 0, \quad t \in[0, T],
$$

where $h: \mathbb{R}^{n} \times \mathbb{R}^{r} \rightarrow \mathbb{R}$ is a given function. For simplicity, we assume that there is only one such constraint. However, the subsequent results can be extended in a straightforward manner to problems with multiple constraints. Let $\mathcal{F}$ denote the set of all $\mathbf{u} \in \mathcal{U}$ satisfying (2.3) almost everywhere. Such controls are called feasible controls. We state our optimal control problem formally as follows.

Problem P. Choose a feasible control $\mathbf{u} \in \mathcal{F}$ that minimizes the cost functional

$$
J(\mathbf{u}):=\Phi(\mathbf{x}(T \mid \mathbf{u})),
$$

where $\Phi: \mathbb{R}^{n} \rightarrow \mathbb{R}$, over $\mathcal{F}$.

Assumption 2.3. The functions $h$ and $\Phi$ are continuously differentiable.

\section{Problem approximation}

In general, Problem $\mathrm{P}$ is too complicated to solve analytically. Thus, in this section, we will derive a class of simpler approximate problems corresponding to Problem P. More specifically, the admissible controls will be restricted to suitable piecewise constant functions, and this produces a sequence of finite-dimensional optimization problems approximating Problem P. Convergence results linking Problem $\mathrm{P}$ with these approximate problems will be given later. 
Let $p \geq 1$ be a fixed integer and define

$$
\begin{aligned}
\Gamma^{p}:=\left\{\boldsymbol{\tau} \in \mathbb{R}^{p+1}: \tau_{0}=0 ;\right. & \tau_{p}=T \\
\tau_{i-1} & \left.\leq \tau_{i}, i=1, \ldots, p\right\} .
\end{aligned}
$$

Moreover, let $\Xi^{p}$ be the set of all $\boldsymbol{\sigma}=\left(\boldsymbol{\sigma}^{1}, \ldots, \boldsymbol{\sigma}^{p}\right)$ such that $\sigma^{i} \in \Upsilon, i=1, \ldots, p$. We consider piecewise constant controls of the form

$$
\mathbf{u}^{p}(t \mid \boldsymbol{\sigma}, \boldsymbol{\tau})=\sum_{i=1}^{p} \boldsymbol{\sigma}^{i} \chi_{\mathcal{I}_{i}^{p}(\boldsymbol{\tau})}(t), \quad t \in[0, T],
$$

where $\boldsymbol{\sigma} \in \Xi^{p}, \boldsymbol{\tau} \in \Gamma^{p}, \mathcal{I}_{i}^{p}(\boldsymbol{\tau})=\left[\tau_{i-1}, \tau_{i}\right)$ (or $\left[\tau_{i-1}, \tau_{i}\right]$ if $i=p$ ), and $\chi_{\mathcal{I}}$ is the characteristic function of the interval $\mathcal{I} \subset \mathbb{R}$. Clearly, $\mathbf{u}^{p}(\cdot \mid \boldsymbol{\sigma}, \boldsymbol{\tau}) \in \mathcal{U}$. The time points $\tau_{i}$, $i=1, \ldots, p-1$, are called switching times for the piecewise constant control.

If the admissible controls are restricted to those of the form (3.1), then the dynamics (2.1) become

$$
\dot{\mathbf{x}}(t)=\sum_{i=1}^{p} \mathbf{f}\left(\mathbf{x}(t), \boldsymbol{\sigma}^{i}\right) \chi_{\mathcal{I}_{i}^{p}(\boldsymbol{\tau})}(t), \quad t \in[0, T] .
$$

The initial condition (2.2) remains the same. Let $\mathbf{x}^{p}(\cdot \mid \boldsymbol{\sigma}, \boldsymbol{\tau})$ denote the solution of (3.2) and (2.2) corresponding to $(\boldsymbol{\sigma}, \boldsymbol{\tau}) \in \Xi^{p} \times \Gamma^{p}$. That is,

$$
\mathbf{x}^{p}(\cdot \mid \boldsymbol{\sigma}, \boldsymbol{\tau})=\mathbf{x}\left(\cdot \mid \mathbf{u}^{p}(\cdot \mid \boldsymbol{\sigma}, \boldsymbol{\tau})\right) .
$$

Hence, with the controls restricted to the form specified by (3.1), the constraint (2.3) becomes

$$
h\left(\mathbf{x}^{p}(t \mid \boldsymbol{\sigma}, \boldsymbol{\tau}), \boldsymbol{\sigma}^{i}\right) \geq 0, \quad t \in \mathcal{I}_{i}^{p}(\boldsymbol{\tau}), \quad i=1, \ldots, p .
$$

Let $\Omega^{p}$ denote the set of all pairs $(\boldsymbol{\sigma}, \boldsymbol{\tau}) \in \Xi^{p} \times \Gamma^{p}$ satisfying the constraint (3.3) everywhere except possibly at $t=T$. Note that

$$
\Omega^{p}=\left\{(\boldsymbol{\sigma}, \boldsymbol{\tau}) \in \Xi^{p} \times \Gamma^{p}: \mathbf{u}^{p}(\cdot \mid \boldsymbol{\sigma}, \boldsymbol{\tau}) \in \mathcal{F}\right\} .
$$

We define an approximate problem as follows.

Problem $\mathbf{P}^{p}$. Choose a pair $(\boldsymbol{\sigma}, \boldsymbol{\tau}) \in \Omega^{p}$ that minimizes the cost function

$$
J^{p}(\boldsymbol{\sigma}, \boldsymbol{\tau}):=J\left(\mathbf{u}^{p}(\cdot \mid \boldsymbol{\sigma}, \boldsymbol{\tau})\right)
$$

over $\Omega^{p}$

Remark 3.1. If $\left(\boldsymbol{\sigma}^{*}, \boldsymbol{\tau}^{*}\right)$ is a solution of Problem $\mathrm{P}^{p}$, then $\mathbf{u}^{p}\left(\cdot \mid \boldsymbol{\sigma}^{*}, \boldsymbol{\tau}^{*}\right)$ is a suboptimal control for Problem P.

Remark 3.2. Here, we have used piecewise constant basis functions to approximate the control. Other basis functions can be used if a continuous control function is required (for example, piecewise linear basis functions).

\section{Transforming the approximate problems}

For each integer $p \geq 1$, Problem $\mathrm{P}^{p}$ is an optimization problem in which the switching times and heights for an approximate piecewise constant control are decision variables to be chosen optimally. To solve these problems using a conventional optimization technique, the gradient of the cost function is required. It has been shown, however, that the gradient of the cost function with respect to the switching times is not useful for numerical computation (Teo, Jennings, Lee, \& Rehbock (1998)). It is also difficult to integrate the system (3.2) and (2.2) accurately if some of the subintervals $\mathcal{I}_{i}^{p}(\boldsymbol{\tau}), i=1, \ldots, p$, are very short. To overcome these two difficulties, a novel transformation will be applied to Problem $\mathrm{P}^{p}$.

Let $p \geq 1$ be a fixed integer and define the set

$$
\begin{aligned}
\Theta^{p}:=\left\{\boldsymbol{\theta} \in \mathbb{R}^{p}: \theta_{i} \geq 0\right. & , i=1, \ldots, p \\
& \left.\theta_{1}+\cdots+\theta_{p}=T\right\} .
\end{aligned}
$$

For each $\boldsymbol{\theta}=\left[\theta_{1}, \ldots, \theta_{p}\right]^{T} \in \Theta^{p}$, define a corresponding function $\nu_{p}(\cdot \mid \boldsymbol{\theta}):[0,1] \rightarrow \mathbb{R}$ as follows:

$\nu_{p}(s \mid \boldsymbol{\theta}):= \begin{cases}\sum_{k=1}^{\lfloor p s\rfloor} \theta_{k}+\theta_{\lfloor p s\rfloor+1}(p s-\lfloor p s\rfloor), & \text { if } s \in[0,1), \\ T, & \text { if } s=1,\end{cases}$

where $\lfloor\cdot\rfloor$ denotes the floor function. Note that $\nu_{p}(\cdot \mid \boldsymbol{\theta})$ is continuous and non-decreasing.

Now, for each $\boldsymbol{\theta} \in \Theta^{p}$, define the following vector:

$$
\boldsymbol{\tau}^{p}(\boldsymbol{\theta}):=\left[\tau_{0}^{p}(\boldsymbol{\theta}), \ldots, \tau_{p}^{p}(\boldsymbol{\theta})\right]^{T} \in \mathbb{R}^{p+1},
$$

where

$$
\tau_{i}^{p}(\boldsymbol{\theta}):=\nu_{p}(i / p \mid \boldsymbol{\theta})=\sum_{k=1}^{i} \theta_{k}, \quad i=0, \ldots, p .
$$

Since $\nu_{p}(\cdot \mid \boldsymbol{\theta})$ is non-decreasing, $\boldsymbol{\tau}^{p}(\boldsymbol{\theta}) \in \Gamma^{p}$. In fact, using (4.1), it is not difficult to see that

$$
\Gamma^{p}=\left\{\boldsymbol{\tau}^{p}(\boldsymbol{\theta}): \boldsymbol{\theta} \in \Theta^{p}\right\}
$$

Furthermore, since $\nu_{p}(\cdot \mid \boldsymbol{\theta})$ is continuous as well as monotonic, it constitutes a surjective mapping from $[0,1]$ to $[0, T]$. On this basis, for each $(\boldsymbol{\sigma}, \boldsymbol{\theta}) \in \Xi^{p} \times \Theta^{p}$, we can define a new state variable

$$
\tilde{\mathbf{x}}^{p}(s \mid \boldsymbol{\sigma}, \boldsymbol{\theta}):=\mathbf{x}^{p}\left(\nu_{p}(s \mid \boldsymbol{\theta}) \mid \boldsymbol{\sigma}, \boldsymbol{\tau}^{p}(\boldsymbol{\theta})\right), \quad s \in[0,1] .
$$


Clearly,

$$
\dot{\nu}_{p}(s \mid \boldsymbol{\theta})=p \theta_{i}, \quad s \in \mathcal{J}_{i}^{p}, \quad i=1, \ldots, p,
$$

where

$$
\mathcal{J}_{i}^{p}:=\left(\frac{i-1}{p}, \frac{i}{p}\right) .
$$

Hence, from (2.2), (3.2), and (4.3)-(4.4), we have

$$
\begin{aligned}
& \dot{\mathbf{x}}^{p}(s \mid \boldsymbol{\sigma}, \boldsymbol{\theta})=p \theta_{i} \mathbf{f}\left(\tilde{\mathbf{x}}^{p}(s \mid \boldsymbol{\sigma}, \boldsymbol{\theta}), \boldsymbol{\sigma}^{i}\right), \\
& s \in \mathcal{J}_{i}^{p}, \quad i=1, \ldots, p,
\end{aligned}
$$

with

$$
\tilde{\mathbf{x}}^{p}(i / p \mid \boldsymbol{\sigma}, \boldsymbol{\theta})=\lim _{s \rightarrow i / p} \tilde{\mathbf{x}}^{p}(s \mid \boldsymbol{\sigma}, \boldsymbol{\theta}), i=0, \ldots, p,
$$

and

$$
\tilde{\mathbf{x}}^{p}(0 \mid \boldsymbol{\sigma}, \boldsymbol{\theta})=\mathbf{x}^{0} .
$$

Now, let $\Lambda^{p}$ denote the set of all pairs $(\boldsymbol{\sigma}, \boldsymbol{\theta}) \in \Xi^{p} \times \Theta^{p}$ satisfying the following constraints:

$$
\theta_{i} h\left(\tilde{\mathbf{x}}^{p}(s \mid \boldsymbol{\sigma}, \boldsymbol{\theta}), \boldsymbol{\sigma}^{i}\right) \geq 0, \quad s \in \overline{\mathcal{J}}_{i}^{p}, \quad i=1, \ldots, p,
$$

where the overhead bar denotes set closure. We have the following important result. Since the proof is tedious, we relegate it to an appendix.

Theorem 4.1. Let $(\boldsymbol{\sigma}, \boldsymbol{\theta}) \in \Xi^{p} \times \Theta^{p}$. Then $(\boldsymbol{\sigma}, \boldsymbol{\theta}) \in \Lambda^{p}$ if and only if $\left(\boldsymbol{\sigma}, \boldsymbol{\tau}^{p}(\boldsymbol{\theta})\right) \in \Omega^{p}$.

Now, a new optimization problem is defined as follows.

Problem $\tilde{\mathbf{P}}^{p}$. Choose $(\boldsymbol{\sigma}, \boldsymbol{\theta}) \in \Lambda^{p}$ to minimize the cost function

$$
\tilde{J}^{p}(\boldsymbol{\sigma}, \boldsymbol{\theta}):=J^{p}\left(\boldsymbol{\sigma}, \boldsymbol{\tau}^{p}(\boldsymbol{\theta})\right)=\Phi\left(\tilde{\mathbf{x}}^{p}(1 \mid \boldsymbol{\sigma}, \boldsymbol{\theta})\right)
$$

over $\Lambda^{p}$.

Remark 4.1. Problems $\mathrm{P}^{p}$ and $\tilde{\mathrm{P}}^{p}$ are equivalent. Indeed, it can be shown from Theorem 4.1 and equation (4.2) that $\left(\boldsymbol{\sigma}^{*}, \boldsymbol{\theta}^{*}\right) \in \Lambda^{p}$ is optimal for Problem $\tilde{\mathrm{P}}^{p}$ if and only if $\left(\boldsymbol{\sigma}^{*}, \boldsymbol{\tau}^{p}\left(\boldsymbol{\theta}^{*}\right)\right) \in \Omega^{p}$ is optimal for Problem $\mathrm{P}^{p}$.

Remark 4.2. Note that the switching times for the dynamic system (4.5)-(4.7) are not decision variables in Problem $\tilde{\mathrm{P}}^{p}$, and therefore do not change as the problem is being solved. In fact, since the sets $\overline{\mathcal{J}}_{i}^{p}, i=1, \ldots, p$, are fixed, Problem $\tilde{\mathrm{P}}^{p}$ is in a form that can be solved readily using an existing algorithm. This is discussed in the next section.

Remark 4.3. The idea of transforming the time horizon of an optimal control problem was originally proposed by Teo et al. (1998). It has since been applied in several other settings, including optimal control problems involving switched systems (Xu \& Antsaklis (2004)), optimal discrete-valued control problems (Lee, Teo, Rehbock, \& Jennings (1999)), and optimal control problems with non-standard cost and constraint functionals (Loxton, Teo, \& Rehbock (2008)).

Remark 4.4. Suppose that Problem $\tilde{\mathrm{P}}^{p}$ has an optimal solution $\left(\boldsymbol{\sigma}^{*}, \boldsymbol{\theta}^{*}\right) \in \Lambda^{p}$. Then according to Remark 4.1, $\left(\boldsymbol{\sigma}^{*}, \boldsymbol{\tau}^{p}\left(\boldsymbol{\theta}^{*}\right)\right) \in \Omega^{p}$ is optimal for Problem $\mathrm{P}^{p}$. The corresponding suboptimal control for Problem $\mathrm{P}$ is $\mathbf{u}^{p}\left(\cdot \mid \boldsymbol{\sigma}^{*}, \boldsymbol{\tau}^{p}\left(\boldsymbol{\theta}^{*}\right)\right)$ (see Remark 3.1 and equation (3.1)).

\section{$5 \quad$ Solving Problem $\tilde{\mathbf{P}}^{p}$}

Since (4.8) are continuous inequality constraints, Problem $\tilde{\mathrm{P}}^{p}$ can be viewed as a semi-infinite programming problem. An efficient algorithm for solving optimization problems of this type is discussed by Teo, Rehbock, \& Jennings (1993). We will now briefly discuss the application of this algorithm to Problem $\tilde{\mathrm{P}}^{p}$. First, let $p \geq 1$ be fixed, and for each $i=1, \ldots, p$, define

$$
\tilde{g}_{i, \epsilon}^{p}(\boldsymbol{\sigma}, \boldsymbol{\theta}):=\int_{\frac{i-1}{p}}^{\frac{i}{p}} \phi_{\epsilon}\left(\theta_{i} h\left(\tilde{\mathbf{x}}^{p}(s \mid \boldsymbol{\sigma}, \boldsymbol{\theta}), \boldsymbol{\sigma}^{i}\right)\right) d s,
$$

where $\epsilon>0$ and

$$
\phi_{\epsilon}(\eta)= \begin{cases}\eta, & \text { if } \eta<-\epsilon, \\ -(\eta-\epsilon)^{2} / 4 \epsilon, & \text { if }-\epsilon \leq \eta \leq \epsilon, \\ 0, & \text { otherwise }\end{cases}
$$

Next, consider the following auxiliary problem.

Problem $\tilde{\mathbf{P}}_{\epsilon, \vartheta}^{p}$. Choose a pair $(\boldsymbol{\sigma}, \boldsymbol{\theta}) \in \Xi^{p} \times \Theta^{p}$ to minimize the cost function

$$
\tilde{G}_{\epsilon, \vartheta}^{p}(\boldsymbol{\sigma}, \boldsymbol{\theta}):=\tilde{J}^{p}(\boldsymbol{\sigma}, \boldsymbol{\theta})-\vartheta \sum_{i=1}^{p} \tilde{g}_{i, \epsilon}^{p}(\boldsymbol{\sigma}, \boldsymbol{\theta}),
$$

where $\epsilon>0$ and $\vartheta>0$ are fixed, over $\Xi^{p} \times \Theta^{p}$.

Each of these auxiliary problems is a nonlinear optimization problem with a single linear equality constraint (recall the definition of $\Theta^{p}$ ) and simple bounds on the variables. Computing the gradient of the linear constraint is straightforward. Furthermore, the gradient of the cost function $\tilde{G}_{\epsilon, \vartheta}^{p}$ can be computed according to the formulae reported in Teo et al. (1991). Hence, Problem $\tilde{\mathrm{P}}_{\epsilon, \vartheta}^{p}$ can be solved efficiently using a gradient-based optimization technique.

The relationship between Problems $\tilde{\mathrm{P}}_{\epsilon, \vartheta}^{p}$ and Problem $\tilde{\mathrm{P}}^{p}$ is furnished in the following two theorems. Proofs of these results are given in Teo et al. (1993). 
Theorem 5.1. For each $\epsilon>0$, there exists a corresponding $\vartheta(\epsilon)>0$ such that for all $\vartheta>\vartheta(\epsilon)$, the optimal solution of Problem $\tilde{\mathrm{P}}_{\epsilon, \vartheta}^{p}$ is feasible for Problem $\tilde{\mathrm{P}}^{p}$.

Theorem 5.2. Suppose that Problem $\tilde{\mathrm{P}}^{p}$ has an optimal solution $\left(\boldsymbol{\sigma}^{*}, \boldsymbol{\theta}^{*}\right) \in \Xi^{p} \times \Theta^{p}$. For each $\epsilon>0$, let $\left(\boldsymbol{\sigma}_{\epsilon, \vartheta}^{*}, \boldsymbol{\theta}_{\epsilon, \vartheta}^{*}\right)$ denote the solution of Problem $\tilde{\mathrm{P}}_{\epsilon, \vartheta}^{p}$, where $\vartheta>0$ is chosen to ensure that $\left(\boldsymbol{\sigma}_{\epsilon, \vartheta}^{*}, \boldsymbol{\theta}_{\epsilon, \vartheta}^{*}\right) \in \Lambda^{p}$ (Theorem 5.1 guarantees that this can always be done). Then

$$
\lim _{\epsilon \rightarrow 0} \tilde{J}^{p}\left(\boldsymbol{\sigma}_{\epsilon, \vartheta}^{*}, \boldsymbol{\theta}_{\epsilon, \vartheta}^{*}\right)=\tilde{J}^{p}\left(\boldsymbol{\sigma}^{*}, \boldsymbol{\theta}^{*}\right) .
$$

Theorems 5.1 and 5.2 suggest the following algorithm for solving Problem $\tilde{\mathrm{P}}^{p}$. First, choose an initial positive value for $\epsilon$. Then, repeatedly solve Problem $\tilde{\mathrm{P}}_{\epsilon, \vartheta}^{p}$ for increasing values of $\vartheta$ until the solution obtained is feasible for Problem $\tilde{\mathrm{P}}^{p}$. According to Theorem 5.1, this process will eventually terminate after a finite number of iterations. Next, we decrease $\epsilon$ and repeat the above procedure, using the solution obtained in the previous step as the new starting point. The algorithm terminates when $\epsilon$ is sufficiently small. It follows from Theorem 5.2 that the solution of Problem $\tilde{\mathrm{P}}_{\epsilon, \vartheta}^{p}$ at this stage is a good approximation of the solution of Problem $\tilde{\mathrm{P}}^{p}$.

Remark 5.1. This procedure solves Problem $\tilde{\mathrm{P}}^{p}$ as a sequence of linearly-constrained optimization problems. These problems can be solved efficiently, because the feasible region is convex and the gradient of the cost function can be computed. Nevertheless, since the cost function is not necessarily convex, it may be difficult to find a global solution. Accordingly, it may be necessary to employ a specialized global optimization technique or start the optimization from multiple starting points when solving Problem $\tilde{\mathrm{P}}_{\epsilon, \vartheta}^{p}$.

\section{Convergence of the suboptimal controls}

For each $p \geq 1$, Problem $\tilde{\mathrm{P}}^{p}$ can be solved efficiently using the penalty function algorithm discussed in the previous section. Then, a suboptimal control for Problem P can be constructed according to Remark 4.4. Repeating this procedure for increasing values of $p$ yields a sequence of suboptimal controls. The following question arises: Does this sequence converge to an optimal control in some sense? The following result will play a crucial role in answering this question.

Theorem 6.1. If $\mathbf{u} \in \mathcal{U}$, then there exists a sequence $\left\{\left(\boldsymbol{\sigma}^{p}, \boldsymbol{\tau}^{p}\right)\right\}_{p=1}^{\infty}$, where $\left(\boldsymbol{\sigma}^{p}, \boldsymbol{\tau}^{p}\right) \in \Xi^{p} \times \Gamma^{p}$ for each $p$, such that $\mathbf{u}^{p}\left(\cdot \mid \boldsymbol{\sigma}^{p}, \boldsymbol{\tau}^{p}\right) \rightarrow \mathbf{u}$ uniformly almost everywhere on $[0, T]$ as $p \rightarrow \infty$.
Proof. Let $\left\{t_{q}\right\}_{q=0}^{d} \subset[0, T]$, where $t_{0}=0, t_{d}=T$, and $t_{q-1}<t_{q}, q=1, \ldots, d$, be a finite set containing all points of discontinuity of $\mathbf{u} \in \mathcal{U}$. Clearly, for each $q=1, \ldots, d$, the restriction of $\mathbf{u}$ to $\left(t_{q-1}, t_{q}\right)$ is uniformly continuous. Hence, for each $\delta>0$, there is a corresponding $\omega_{\delta}>0$ such that if $q \in\{1, \ldots, d\}$ and

$$
\eta_{1}, \eta_{2} \in\left(t_{q-1}, t_{q}\right),\left\|\eta_{1}-\eta_{2}\right\|<\omega_{\delta},
$$

then

$$
\left\|\mathbf{u}\left(\eta_{1}\right)-\mathbf{u}\left(\eta_{2}\right)\right\|<\delta
$$

Now, let $\left\{\boldsymbol{\tau}^{p}\right\}_{p=1}^{\infty}$ be a sequence of vectors with the following properties:

(i) For each $p \geq 1, \tau^{p} \in \Gamma^{p}$ and $\tau_{i-1}^{p}<\tau_{i}^{p}, i=1, \ldots, p$;

(ii) If $p \geq d$, then for each $q=0, \ldots, d$, there is an integer $\kappa_{p, q}$ such that $\tau_{\kappa_{p, q}}^{p}=t_{q}$; and

(iii) $\max _{1 \leq i \leq p}\left(\tau_{i}^{p}-\tau_{i-1}^{p}\right) \rightarrow 0$ as $p \rightarrow \infty$.

It is easy to envisage such a sequence, but cumbersome to describe it analytically. The important point to note is that each vector after the first $d-1$ terms of the sequence contains the time points $t_{q}, q=0, \ldots, d$, as components (see Property (ii)).

We define another sequence as follows: For each $p \geq 1$, let $\boldsymbol{\sigma}^{p}:=\left(\boldsymbol{\sigma}^{p, 1}, \ldots, \boldsymbol{\sigma}^{p, p}\right)$, where

$$
\boldsymbol{\sigma}^{p, i}:=\frac{1}{\tau_{i}^{p}-\tau_{i-1}^{p}} \int_{\tau_{i-1}^{p}}^{\tau_{i}^{p}} \mathbf{u}(\eta) d \eta, \quad i=1, \ldots, p
$$

Since $\mathbf{u}$ is admissible, each $\boldsymbol{\sigma}^{p, i} \in \Upsilon$, so $\boldsymbol{\sigma}^{p} \in \Xi^{p}$. Hence, $\left(\boldsymbol{\sigma}^{p}, \boldsymbol{\tau}^{p}\right) \in \Xi^{p} \times \Gamma^{p}$ for every integer $p \geq 1$. We will show that $\mathbf{u}^{p}\left(\cdot \mid \boldsymbol{\sigma}^{p}, \boldsymbol{\tau}^{p}\right) \rightarrow \mathbf{u}$ uniformly almost everywhere on $[0, T]$ as $p \rightarrow \infty$

Let $\delta>0$ be arbitrary and suppose that $t \in\left(t_{l-1}, t_{l}\right)$ for some $l \in\{1, \ldots, d\}$. Then for each $p \geq 1$, there is an integer $i_{p} \in\{1, \ldots, p\}$ such that $t \in \mathcal{I}_{i_{p}}^{p}\left(\boldsymbol{\tau}^{p}\right)$. Thus, for each $p \geq 1$,

$$
\begin{aligned}
& \left\|\mathbf{u}^{p}\left(t \mid \boldsymbol{\sigma}^{p}, \boldsymbol{\tau}^{p}\right)-\mathbf{u}(t)\right\|=\left\|\boldsymbol{\sigma}^{p, i_{p}}-\mathbf{u}(t)\right\| \\
& \quad \leq \frac{1}{\tau_{i_{p}}^{p}-\tau_{i_{p}-1}^{p}} \int_{\tau_{i_{p}-1}^{p}}^{\tau_{i_{p}}^{p}}\|\mathbf{u}(\eta)-\mathbf{u}(t)\| d \eta \\
& \quad=\frac{1}{\tau_{i_{p}}^{p}-\tau_{i_{p}-1}^{p}} \int_{\dot{\mathcal{I}}_{i_{p}}^{p}\left(\boldsymbol{\tau}^{p}\right)}\|\mathbf{u}(\eta)-\mathbf{u}(t)\| d \eta,
\end{aligned}
$$

where $\stackrel{\circ}{\mathcal{I}}_{i_{p}}^{p}\left(\boldsymbol{\tau}^{p}\right)$ denotes the interior of $\mathcal{I}_{i_{p}}^{p}\left(\boldsymbol{\tau}^{p}\right)$. Property (iii) above implies the existence of an integer $p_{1} \geq 1$, which depends only on $\delta$, such that

$$
\max _{1 \leq i \leq p}\left(\tau_{i}^{p}-\tau_{i-1}^{p}\right)<\omega_{\delta}, \quad p \geq p_{1},
$$


where $\omega_{\delta}$ is as defined previously. If $p \geq \max \left\{p_{1}, d\right\}$, then it follows from Property (ii) that

$$
\stackrel{\circ}{\mathcal{I}}_{i_{p}}^{p}\left(\boldsymbol{\tau}^{p}\right) \subset\left(t_{l-1}, t_{l}\right)
$$

Moreover, from (6.2), we have

$$
\eta \in{\stackrel{\mathcal{I}}{i_{p}}}_{p}^{p}\left(\boldsymbol{\tau}^{p}\right) \quad \Longrightarrow \quad|\eta-t| \leq \tau_{i_{p}}^{p}-\tau_{i_{p}-1}^{p}<\omega_{\delta} .
$$

Using (6.3), (6.4), and the definition of $\omega_{\delta}$ in (6.1), we obtain

$$
\left\|\mathbf{u}^{p}\left(t \mid \boldsymbol{\sigma}^{p}, \boldsymbol{\tau}^{p}\right)-\mathbf{u}(t)\right\|<\frac{1}{\tau_{i_{p}}^{p}-\tau_{i_{p}-1}^{p}} \int_{\dot{\mathcal{I}}_{i_{p}}^{p}\left(\boldsymbol{\tau}^{p}\right)} \delta=\delta
$$

Since $\delta$ was arbitrary, and $p$ was selected independently of $l$ and $t$, we have shown that $\mathbf{u}^{p}\left(\cdot \mid \boldsymbol{\sigma}^{p}, \boldsymbol{\tau}^{p}\right) \rightarrow \mathbf{u}$ uniformly on $[0, T] \backslash\left\{t_{0}, \ldots, t_{d}\right\}$ as $p \rightarrow \infty$.

Recall the following two results from Chapter 6 of Teo et al. (1991).

Lemma 6.1. There exists a constant $L_{2}>0$ such that

$$
\|\mathbf{x}(t \mid \mathbf{u})\| \leq L_{2}, \quad t \in[0, T], \quad \mathbf{u} \in \mathcal{U}
$$

Lemma 6.2. Let $\left\{\mathbf{u}^{p}\right\}_{p=1}^{\infty} \subset \mathcal{U}$ be a sequence of admissible controls converging to an admissible control $\mathbf{u} \in \mathcal{U}$ almost everywhere on $[0, T]$. Then:

(i) $\mathbf{x}\left(\cdot \mid \mathbf{u}^{p}\right) \rightarrow \mathbf{x}(\cdot \mid \mathbf{u})$ uniformly on $[0, T]$ as $p \rightarrow \infty$.

(ii) $J\left(\mathbf{u}^{p}\right) \rightarrow J(\mathbf{u})$ as $p \rightarrow \infty$.

For convenience, define the set

$$
\Psi:=\left\{\mathbf{x} \in \mathbb{R}^{n}:\|\mathbf{x}\| \leq L_{2}\right\}
$$

where $L_{2}$ is the constant from Lemma 6.1. Furthermore, let $\stackrel{\circ}{\mathcal{F}}$ denote the class of all admissible control functions $\mathbf{u} \in \mathcal{U}$ with the following property: There exists a set $\mathcal{V}$ of measure zero such that

$$
\inf _{t \in[0, T] \backslash \mathcal{V}} h(\mathbf{x}(t \mid \mathbf{u}), \mathbf{u}(t))>0
$$

Notice that controls in $\stackrel{\mathcal{F}}{ }$ satisfy the constraints $(2.3)$ strictly for almost all $t \in[0, T]$. Hence, $\stackrel{\circ}{\mathcal{F}} \subset \mathcal{F}$. We assume that the following condition is satisfied.

Assumption 6.1. If $\mathbf{u}^{*} \in \mathcal{F}$ is an optimal control for Problem $\mathrm{P}$, then there exists a $\overline{\mathbf{u}} \in \mathcal{\mathcal { F }}$ such that

$$
\omega \overline{\mathbf{u}}+(1-\omega) \mathbf{u}^{*} \in \stackrel{\circ}{\mathcal{F}}, \quad \omega \in(0,1]
$$

Similar assumptions are made in Teo \& Jennings (1989), Teo et al. (1991), and Teo et al. (1993). We now derive the following convergence result.

Theorem 6.2. Suppose that $\mathbf{u}^{*}$ is an optimal control for Problem P. For each $p \geq 1$, let $\mathbf{u}^{p \text {,* }}$ denote the suboptimal control constructed from the solution of Problem $\mathrm{P}^{p}$ according to Remark 3.1. Then

$$
\lim _{p \rightarrow \infty} J\left(\mathbf{u}^{p, *}\right)=J\left(\mathbf{u}^{*}\right) .
$$

Proof. By Assumption 6.1, there exists a $\overline{\mathbf{u}} \in \stackrel{\circ}{\mathcal{F}}_{\text {such }}$ that

$$
\overline{\mathbf{u}}^{k}:=\mathbf{u}^{*}+\frac{1}{k}\left(\overline{\mathbf{u}}-\mathbf{u}^{*}\right) \in \stackrel{\circ}{\mathcal{F}}
$$

for every integer $k \geq 1$. Hence, for each $k \geq 1$, there is a corresponding constant $v_{k}>0$ and a corresponding set $\mathcal{V}_{k} \subset[0, T]$ of measure zero such that

$$
h\left(\mathbf{x}\left(t \mid \overline{\mathbf{u}}^{k}\right), \overline{\mathbf{u}}^{k}(t)\right) \geq v_{k}, \quad t \in[0, T] \backslash \mathcal{V}_{k}
$$

Now, fix $k$ and let $\left\{\left(\overline{\boldsymbol{\sigma}}^{k, p}, \overline{\boldsymbol{\tau}}^{k, p}\right)\right\}_{p=1}^{\infty}$ denote the sequence from Theorem 6.1 corresponding to the admissible control $\overline{\mathbf{u}}^{k}$. We write $\overline{\mathbf{u}}^{k, p}=\mathbf{u}^{p}\left(\cdot \mid \overline{\boldsymbol{\sigma}}^{k, p}, \overline{\boldsymbol{\tau}}^{k, p}\right)$ for convenience. Observe the following:

(i) There exists a set $\mathcal{N}_{k} \subset[0, T]$ of measure zero such that $\overline{\mathbf{u}}^{k, p}(t) \in \Upsilon, p \geq 1$, and $\overline{\mathbf{u}}^{k}(t) \in \Upsilon$ for every $t \in[0, T] \backslash \mathcal{N}_{k}$ (definition of $\left.\mathcal{U}\right)$

(ii) There exists a set $\mathcal{G}_{k} \subset[0, T]$ of measure zero such that $\overline{\mathbf{u}}^{k, p} \rightarrow \overline{\mathbf{u}}^{k}$ uniformly on $[0, T] \backslash \mathcal{G}_{k}$ as $p \rightarrow \infty$ (Theorem 6.1);

(iii) $\mathbf{x}\left(\cdot \mid \overline{\mathbf{u}}^{k, p}\right) \rightarrow \mathbf{x}\left(\cdot \mid \overline{\mathbf{u}}^{k}\right)$ uniformly on $[0, T]$ as $p \rightarrow \infty$ (part (i) of Lemma 6.2);

(iv) $\mathbf{x}\left(t \mid \overline{\mathbf{u}}^{k, p}\right) \in \Psi$ and $\mathbf{x}\left(t \mid \overline{\mathbf{u}}^{k}\right) \in \Psi$ for every $t \in[0, T]$ (Lemma 6.1); and

(v) $h$ is uniformly continuous on $\Psi \times \Upsilon$ (Assumption 2.3).

Statements (i)-(v) above imply the existence of an integer $p_{k, 1} \geq 1$ and a set $\mathcal{M}_{k}$ of measure zero such that

$$
\begin{gathered}
\left|h\left(\mathbf{x}\left(t \mid \overline{\mathbf{u}}^{k, p}\right), \overline{\mathbf{u}}^{k, p}(t)\right)-h\left(\mathbf{x}\left(t \mid \overline{\mathbf{u}}^{k}\right), \overline{\mathbf{u}}^{k}(t)\right)\right|<v_{k} / 2, \\
t \in[0, T] \backslash \mathcal{M}_{k},
\end{gathered}
$$

for all $p \geq p_{k, 1}$. Thus, if $p \geq p_{k, 1}$, then it follows from inequalities (6.6) and (6.7) that

$$
h\left(\mathbf{x}\left(t \mid \overline{\mathbf{u}}^{k, p}\right), \overline{\mathbf{u}}^{k, p}(t)\right)>\frac{v_{k}}{2}, t \in[0, T] \backslash\left(\mathcal{M}_{k} \cup \mathcal{V}_{k}\right)
$$

Furthermore, part (ii) of Lemma 6.2 ensures that there exists another positive integer $p_{k, 2} \geq 1$ such that

$$
\left|J\left(\overline{\mathbf{u}}^{k, p}\right)-J\left(\overline{\mathbf{u}}^{k}\right)\right|<\frac{1}{k}
$$


whenever $p \geq p_{k, 2}$. Define $p_{k}:=\max \left\{p_{k, 1}, p_{k, 2}\right\}$. Then since $\mu\left(\mathcal{M}_{k} \cup \mathcal{V}_{k}\right)=0$, inequality (6.8) shows that $\overline{\mathbf{u}}^{k, p_{k}} \in \mathcal{F}$. Hence, from $(3.4),\left(\overline{\boldsymbol{\sigma}}^{k, p_{k}}, \overline{\boldsymbol{\tau}}^{k, p_{k}}\right) \in \Omega^{p_{k}}$.

Now, let $\delta>0$ and recall that $k \geq 1$ was arbitrary. It is readily seen from $(6.5)$ that $\overline{\mathbf{u}}^{k} \rightarrow \mathbf{u}^{*}$ pointwise on $[0, T]$ as $k \rightarrow \infty$. Hence, part (ii) of Lemma 6.2 implies the existence of an integer $k_{1}$ such that

$$
\left|J\left(\overline{\mathbf{u}}^{k}\right)-J\left(\mathbf{u}^{*}\right)\right|<\delta / 2
$$

for all $k \geq k_{1}$. Choose any $k \geq \max \left\{k_{1}, 2 / \delta\right\}$. Then it follows from (6.9) and (6.10) that

$$
\begin{aligned}
&\left|J^{p_{k}}\left(\overline{\boldsymbol{\sigma}}^{k, p_{k}}, \overline{\boldsymbol{\tau}}^{k, p_{k}}\right)-J\left(\mathbf{u}^{*}\right)\right|=\left|J\left(\overline{\mathbf{u}}^{k, p_{k}}\right)-J\left(\mathbf{u}^{*}\right)\right| \\
& \leq\left|J\left(\overline{\mathbf{u}}^{k, p_{k}}\right)-J\left(\overline{\mathbf{u}}^{k}\right)\right|+\left|J\left(\overline{\mathbf{u}}^{k}\right)-J\left(\mathbf{u}^{*}\right)\right| \\
&<\delta .
\end{aligned}
$$

Now, let $p \geq p_{k}$. Then

$$
\begin{aligned}
J\left(\mathbf{u}^{*}\right) & \leq J\left(\mathbf{u}^{p, *}\right) \\
& =J^{p}\left(\boldsymbol{\sigma}^{p, *}, \boldsymbol{\tau}^{p, *}\right) \leq J^{p_{k}}\left(\boldsymbol{\sigma}^{p_{k}, *}, \boldsymbol{\tau}^{p_{k}, *}\right),
\end{aligned}
$$

where $\left(\boldsymbol{\sigma}^{p, *}, \boldsymbol{\tau}^{p, *}\right)$ and $\left(\boldsymbol{\sigma}^{p_{k}, *}, \boldsymbol{\tau}^{p_{k}, *}\right)$ are the optimal solutions for Problems $\mathrm{P}^{p}$ and $\mathrm{P}^{p_{k}}$, respectively. Since $\left(\overline{\boldsymbol{\sigma}}^{k, p_{k}}, \overline{\boldsymbol{\tau}}^{k, p_{k}}\right) \in \Omega^{p_{k}}$, inequality (6.12) gives

$$
J\left(\mathbf{u}^{*}\right) \leq J\left(\mathbf{u}^{p, *}\right) \leq J^{p_{k}}\left(\overline{\boldsymbol{\sigma}}^{k, p_{k}}, \overline{\boldsymbol{\tau}}^{k, p_{k}}\right) .
$$

Finally, applying (6.11) to (6.13) yields

$$
J\left(\mathbf{u}^{*}\right) \leq J\left(\mathbf{u}^{p, *}\right) \leq J^{p_{k}}\left(\overline{\boldsymbol{\sigma}}^{k, p_{k}}, \overline{\boldsymbol{\tau}}^{k, p_{k}}\right)<J\left(\mathbf{u}^{*}\right)+\delta .
$$

Since this estimate holds for each $\delta>0$, it is clear that $J\left(\mathbf{u}^{p, *}\right) \rightarrow J\left(\mathbf{u}^{*}\right)$ as $p \rightarrow \infty$.

Theorem 6.2 shows that the cost of the suboptimal controls converges to the minimum cost as $p \rightarrow \infty$. In general, however, there is no guarantee that the controls themselves converge. Nevertheless, we do have the following important result.

Theorem 6.3. Let $\mathbf{u}^{*}$ and $\mathbf{u}^{p, *}$ be as defined in Theorem 6.2 , and suppose that the sequence $\left\{\mathbf{u}^{p, *}\right\}_{p=1}^{\infty}$ converges almost everywhere on $[0, T]$ to a piecewise continuous function $\hat{\mathbf{u}}:[0, T] \rightarrow \mathbb{R}^{r}$. Then $\hat{\mathbf{u}}$ is an optimal control for Problem P.

Proof. It is easy to verify that $\hat{\mathbf{u}}$ is an admissible control. Furthermore, from part (ii) of Lemma 6.2 we have

$$
\lim _{p \rightarrow \infty} J\left(\mathbf{u}^{p, *}\right)=J(\hat{\mathbf{u}})
$$

This combined with Theorem 6.2 gives $J(\hat{\mathbf{u}})=J\left(\mathbf{u}^{*}\right)$. It remains to show that $\hat{\mathbf{u}}$ is a feasible control. Suppose, to the contrary, that there is a set $\mathcal{C} \subset[0, T]$ of strictly positive measure such that

$$
h(\mathbf{x}(t \mid \hat{\mathbf{u}}), \hat{\mathbf{u}}(t))<0, \quad t \in \mathcal{C} .
$$

For each integer $k \geq 1$, define

$$
\mathcal{W}_{k}:=\{t \in[0, T]: h(\mathbf{x}(t \mid \hat{\mathbf{u}}), \hat{\mathbf{u}}(t)) \leq-1 / k\} .
$$

Clearly, $\left\{\mathcal{W}_{k}\right\}_{k=1}^{\infty}$ is an increasing sequence of measurable sets. Furthermore, it follows from (6.14) that

$$
\mathcal{C} \subset \bigcup_{k=1}^{\infty} \mathcal{W}_{k}
$$

Thus, applying a well-known result in measure theory (see, for example, Halmos (1974)) yields

$$
\mu(\mathcal{C}) \leq \lim _{k \rightarrow \infty} \mu\left(\mathcal{W}_{k}\right)
$$

In particular, we can select an integer $k_{1} \geq 1$ so that

$$
h(\mathbf{x}(t \mid \hat{\mathbf{u}}), \hat{\mathbf{u}}(t)) \leq-1 / k_{1}, \quad t \in \mathcal{W}_{k_{1}}
$$

and

$$
0<\mu(\mathcal{C}) / 2 \leq \mu\left(\mathcal{W}_{k_{1}}\right) .
$$

Now, by Egoroff's Theorem, there is a measurable set $\mathcal{D} \subset[0, T]$, with measure $\mu(\mathcal{D})<\mu\left(\mathcal{W}_{k_{1}}\right) / 2$, such that $\mathbf{u}^{p, *}(t) \in \Upsilon, p \geq 1$, and $\hat{\mathbf{u}}(t) \in \Upsilon$ for each $t \notin \mathcal{D}$, and $\mathbf{u}^{p, *} \rightarrow \hat{\mathbf{u}}$ uniformly on $[0, T] \backslash \mathcal{D}$ as $p \rightarrow \infty$. Thus, recalling that $h$ is uniformly continuous on $\Psi \times \Upsilon$ and noting from part (i) of Lemma 6.2 that $\mathbf{x}\left(\cdot \mid \mathbf{u}^{p, *}\right) \rightarrow \mathbf{x}(\cdot \mid \hat{\mathbf{u}})$ uniformly on $[0, T]$ as $p \rightarrow \infty$, we deduce the existence of an integer $p_{1} \geq 1$ such that

$$
\begin{array}{r}
\left|h\left(\mathbf{x}\left(t \mid \mathbf{u}^{p_{1}, *}\right), \mathbf{u}^{p_{1}, *}(t)\right)-h(\mathbf{x}(t \mid \hat{\mathbf{u}}), \hat{\mathbf{u}}(t))\right|<1 /\left(2 k_{1}\right), \\
t \in[0, T] \backslash \mathcal{D} .
\end{array}
$$

Combining (6.15) and (6.17) gives

$$
h\left(\mathbf{x}\left(t \mid \mathbf{u}^{p_{1}, *}\right), \mathbf{u}^{p_{1}, *}(t)\right)<-\frac{1}{2 k_{1}}, \quad t \in \mathcal{W}_{k_{1}} \backslash \mathcal{D} .
$$

Finally, note that

$$
\begin{aligned}
\mu\left(\mathcal{W}_{k_{1}} \backslash \mathcal{D}\right) & =\mu\left(\mathcal{W}_{k_{1}}\right)-\mu\left(\mathcal{W}_{k_{1}} \cap \mathcal{D}\right) \\
& \geq \mu\left(\mathcal{W}_{k_{1}}\right)-\mu(\mathcal{D})>\mu\left(\mathcal{W}_{k_{1}}\right) / 2>0
\end{aligned}
$$

where the last inequality is a consequence of (6.16). Since $\mathcal{W}_{k_{1}} \backslash \mathcal{D}$ is a set of positive measure, inequality (6.18) contradicts the feasibility of $\mathbf{u}^{p_{1}, *}$. Therefore, $\hat{\mathbf{u}} \in \mathcal{F}$ as required.

Remark 6.1. Theorems 6.2 and 6.3 provide the theoretical justification for solving Problem $\mathrm{P}$ as follows. 
First, choose an initial $p \geq 1$ and solve the corresponding Problem $\tilde{\mathrm{P}}^{p}$ using the procedure developed in Section 5. Then, increase the value of $p$ and, using the optimal solution from the previous step as the starting point, resolve Problem $\tilde{\mathrm{P}}^{p}$. This process can be repeated until the change in the optimal value of the cost function is within a desired tolerance. A suboptimal control for Problem $\mathrm{P}$ can then be constructed according to Remark 4.4.

\section{An example-Rayleigh's problem}

The following problem is from [5]: Choose a control function $u:[0,4.5] \rightarrow \mathbb{R}$ to minimize

$$
\int_{0}^{4.5}\left(u^{2}(t)+x_{1}^{2}(t)\right) d t
$$

subject to the dynamics

$$
\begin{aligned}
& \dot{x}_{1}(t)=x_{2}(t), \\
& \dot{x}_{2}(t)=-x_{1}(t)+x_{2}(t)\left(1.4-0.14 x_{2}^{2}(t)\right)+4 u(t),
\end{aligned}
$$

and

$$
\begin{aligned}
& x_{1}(0)=-5, \\
& x_{2}(0)=-5,
\end{aligned}
$$

and the continuous constraint

$$
-u(t)-\frac{1}{6} x_{1}(t) \geq 0, \quad t \in[0,4.5]
$$

Problems $\tilde{\mathrm{P}}_{\epsilon, \vartheta}^{p}$ corresponding to this optimal control problem can be constructed according to the definitions given in Sections 4 and 5. Furthermore, each of these approximate problems can be solved conveniently using the software MISER (Jennings, Teo, Fisher, \& Goh (2004)). MISER uses the gradient formulae from Teo et al. (1991) in conjunction with the nonlinear programming routine NLPQLP (Schittkowski (2007)) to solve dynamic optimization problems numerically.

Choosing $p=10$ for the number of subintervals, we used MISER to solve a sequence of Problems $\tilde{\mathrm{P}}_{\epsilon, \vartheta}^{p}$. The smoothing and penalty parameters were initially selected as $\epsilon=0.1$ and $\vartheta=10.0$, and then subsequently adjusted according to the guidelines in Section 5. Recall that for each value of $\epsilon$, the penalty parameter $\vartheta$ is increased until the solution obtained is feasible for the original problem. The process was terminated when $\epsilon=1.0 \times 10^{-6}$ and $\vartheta=1.0 \times 10^{5}$. It is worth mentioning that in the first step, a large value of $\vartheta$ was required to ensure feasibility. After that, $\vartheta$ hardly changed as $\epsilon$ was decreased.

The suboptimal control generated from the final solution of Problem $\tilde{\mathrm{P}}_{\epsilon, \vartheta}^{p}$ (see Remark 4.4) is shown along with its corresponding state trajectory and constraint profile in Figure 7.1. The important thing to note is that the control-state constraint is satisfied everywhere. Furthermore, the structure of the suboptimal control agrees with the results obtained in Gerdts (2008a). The major difference is that the method from Gerdts (2008a) gives the optimal control as a continuous function, whereas ours is based on a piecewise constant approximation. Note also that only a small improvement (less than 1\%) was obtained by re-solving the problem with $p=20$.

\section{Conclusion}

In this paper, we have discussed a new computational method for solving nonlinear optimal control problems with a continuous inequality constraint. Our method is a significant generalization of the $\epsilon-\tau$ algorithm reported by Teo \& Jennings (1989) and Teo et al. (1991). In particular, it is capable of handling continuous constraints that include the control function explicitly. The $\epsilon-\tau$ algorithm is not guaranteed to converge for this case.

Since the constraint functions are potentially discontinuous in time, establishing the convergence results of Section 6 was difficult. Indeed, continuity with respect to time was exploited by Teo \& Jennings (1989) and Teo et al. (1991) to prove some important properties of the $\epsilon-\tau$ algorithm. Nevertheless, Theorem 6.2 guarantees that the cost of the suboptimal controls converges to the optimal cost. Furthermore, whilst it is not possible to show that the sequence of suboptimal controls converges to an admissible control, Theorem 6.3 ensures that if it does, then the limit function must be an optimal control.

We must point out that the admissible controls here are restricted to bounded piecewise continuous functions. In Teo \& Jennings (1989) and Teo et al. (1991), the controls were selected from a larger class consisting of bounded measurable functions. The arguments used to establish Theorem 6.1 (and therefore Theorem 6.2) are not valid in this general setting. Nevertheless, almost every realistic control input is piecewise continuous.

\section{Appendix}

Proof of Theorem 4.1. Since $(\boldsymbol{\sigma}, \boldsymbol{\theta}) \in \Xi^{p} \times \Theta^{p}$ is fixed, we denote $\mathbf{x}^{p}\left(\cdot \mid \boldsymbol{\sigma}, \boldsymbol{\tau}^{p}(\boldsymbol{\theta})\right)$ by $\mathbf{x}^{p}(\cdot)$ and $\tilde{\mathbf{x}}^{p}(\cdot \mid \boldsymbol{\sigma}, \boldsymbol{\theta})$ by $\tilde{\mathbf{x}}^{p}(\cdot)$ without confusion. Moreover, define the index sets

$$
\mathcal{S}_{1}:=\left\{i \in\{1, \ldots, p\}: \theta_{i}>0\right\}
$$

and

$$
\mathcal{S}_{2}:=\{1, \ldots, p\} \backslash \mathcal{S}_{1} .
$$

From equation (4.1), we see that

$$
\tau_{i}^{p}(\boldsymbol{\theta})-\tau_{i-1}^{p}(\boldsymbol{\theta})=\theta_{i}, \quad i=1, \ldots, p .
$$



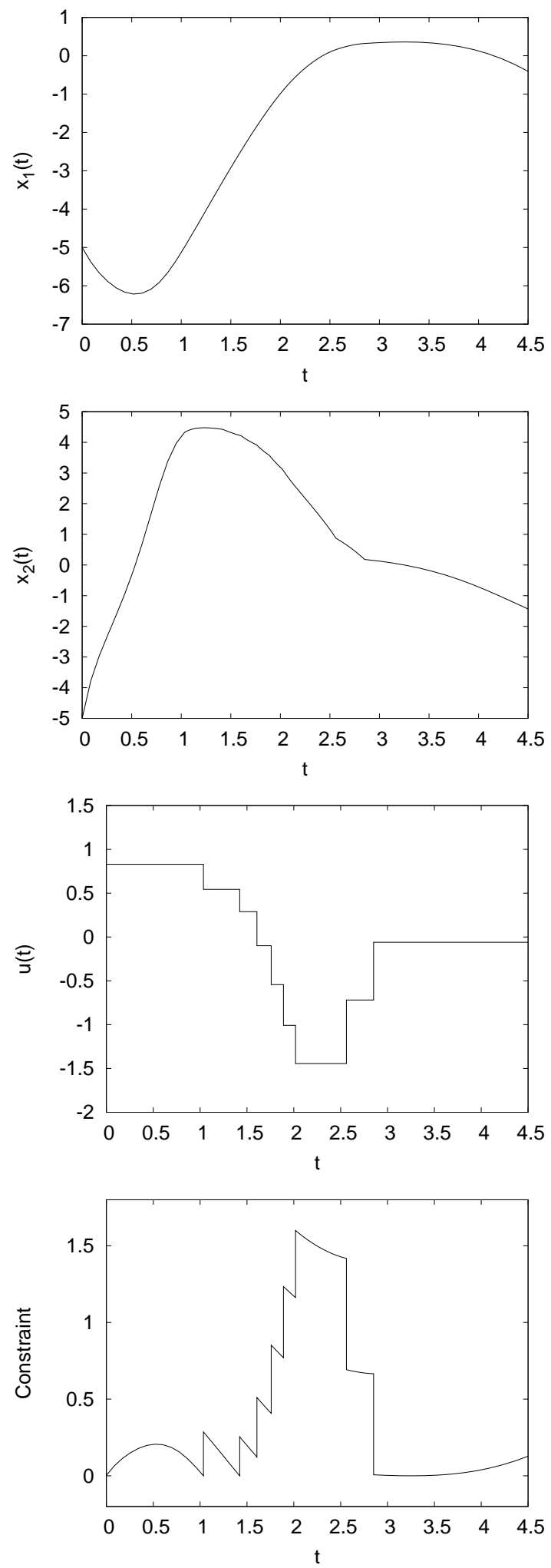

Fig. 7.1. The optimal state trajectory, optimal control profile, and the corresponding continuous constraint profile for Example 7.1.
Therefore,

$$
\tau_{i}^{p}(\boldsymbol{\theta})=\tau_{i-1}^{p}(\boldsymbol{\theta}) \quad \Longleftrightarrow \quad i \in \mathcal{S}_{2} .
$$

Now, suppose $(\boldsymbol{\sigma}, \boldsymbol{\theta}) \in \Lambda^{p}$. Then

$$
\theta_{i} h\left(\tilde{\mathbf{x}}^{p}(s), \boldsymbol{\sigma}^{i}\right) \geq 0, \quad s \in \overline{\mathcal{J}}_{i}^{p}, \quad i=1, \ldots, p .
$$

First, we consider the case $i \in \mathcal{S}_{1}$. Inequalities (A.2) give

$$
h\left(\tilde{\mathbf{x}}^{p}(s), \boldsymbol{\sigma}^{i}\right) \geq 0, \quad s \in \overline{\mathcal{J}}_{i}^{p}, \quad i \in \mathcal{S}_{1} .
$$

Furthermore, $\nu_{p, i}(\cdot \mid \boldsymbol{\theta})$, the restriction of $\nu_{p}(\cdot \mid \boldsymbol{\theta})$ to $\overline{\mathcal{J}}_{i}^{p}$, is a bijective mapping with range $\overline{\mathcal{I}}_{i}^{p}\left(\boldsymbol{\tau}^{p}(\boldsymbol{\theta})\right)$. Consequently, $\nu_{p, i}^{-1}(t \mid \boldsymbol{\theta}) \in \overline{\mathcal{J}}_{i}^{p}$ whenever $t \in \mathcal{I}_{i}^{p}\left(\boldsymbol{\tau}^{p}(\boldsymbol{\theta})\right)$. This fact, together with (A.3), implies

$$
\begin{aligned}
h\left(\mathbf{x}^{p}(t), \boldsymbol{\sigma}^{i}\right)=h\left(\tilde{\mathbf{x}}^{p}\left(\nu_{p, i}^{-1}(t \mid \boldsymbol{\theta})\right), \boldsymbol{\sigma}^{i}\right) \geq 0, \\
\quad t \in \mathcal{I}_{i}^{p}\left(\boldsymbol{\tau}^{p}(\boldsymbol{\theta})\right), \quad i \in \mathcal{S}_{1} .
\end{aligned}
$$

Next, consider $i \in \mathcal{S}_{2}$. If $i=p$, then $\mathcal{I}_{i}^{p}\left(\boldsymbol{\tau}^{p}(\boldsymbol{\theta})\right)=\{T\}$. Hence, it follows from the definition of $\Omega^{p}$ that we only need to consider $i \in \mathcal{S}_{2} \backslash\{p\}$. In this case, it is easy to see from (A.1) that the constraints

$$
h\left(\mathbf{x}^{p}(t), \boldsymbol{\sigma}^{i}\right) \geq 0, t \in \mathcal{I}_{i}^{p}\left(\boldsymbol{\tau}^{p}(\boldsymbol{\theta})\right), i \in \mathcal{S}_{2} \backslash\{p\},
$$

are satisfied vacuously. Inequalities (A.4) and (A.5) show that $\left(\boldsymbol{\sigma}, \boldsymbol{\tau}^{p}(\boldsymbol{\theta})\right) \in \Omega^{p}$.

Conversely, suppose that $\left(\boldsymbol{\sigma}, \boldsymbol{\tau}^{p}(\boldsymbol{\theta})\right) \in \Omega^{p}$. Then

$$
h\left(\mathbf{x}^{p}(t), \boldsymbol{\sigma}^{i}\right) \geq 0, t \in \mathcal{I}_{i}^{p}\left(\boldsymbol{\tau}^{p}(\boldsymbol{\theta})\right), i=1, \ldots, p .
$$

Clearly,

$$
\theta_{i} h\left(\tilde{\mathbf{x}}^{p}(s), \boldsymbol{\sigma}^{i}\right)=0, \quad s \in \overline{\mathcal{J}}_{i}^{p}, \quad i \in \mathcal{S}_{2} .
$$

Furthermore, it follows from (A.1) that $\mathcal{I}_{i}^{p}\left(\boldsymbol{\tau}^{p}(\boldsymbol{\theta})\right) \neq \emptyset$ for $i \in \mathcal{S}_{1}$. Hence, if $s \in \overline{\mathcal{J}}_{i}^{p} \backslash\{i / p\}$, then $\nu_{p}(s \mid \boldsymbol{\theta}) \in$ $\mathcal{I}_{i}^{p}\left(\boldsymbol{\tau}^{p}(\boldsymbol{\theta})\right)$. Thus, the inequalities in (A.6) yield

$$
\begin{array}{r}
\theta_{i} h\left(\tilde{\mathbf{x}}^{p}(s), \boldsymbol{\sigma}^{i}\right)=\theta_{i} h\left(\mathbf{x}^{p}\left(\nu_{p}(s \mid \boldsymbol{\theta})\right), \boldsymbol{\sigma}^{i}\right) \geq 0, \\
s \in \overline{\mathcal{J}}_{i}^{p} \backslash\{i / p\}, \quad i \in \mathcal{S}_{1} .
\end{array}
$$

Finally, since the functions $\tilde{\mathbf{x}}^{p}(\cdot)$, and $h$ are continuous, inequality (A.8) also holds at $s=i / p$. Hence, (A.7) and (A.8) imply $(\boldsymbol{\sigma}, \boldsymbol{\theta}) \in \Lambda^{p}$.

\section{References}

[1] Ahmed, N. U. (2006). Dynamic systems and control with applications. Singapore: World Scientific. 
[2] Büskens, C. \& Maurer, H. (2000). SQP-methods for solving optimal control problems with control and state constraints: adjoint variables, sensitivity analysis and real-time control. Journal of Computational and Applied Mathematics, 120(1), 85-108.

[3] Chen, T. W. C. \& Vassiliadis, V. S. (2005). Inequality path constraints in optimal control: a finite iteration $\varepsilon$-convergent scheme based on pointwise discretization. Journal of Process Control, 15(3), 353-362.

[4] Gates, D. J. \& Westcott, M. (1996). Solar cars and variational problems equivalent to shortest paths. SIAM Journal on Control and Optimization, 34(2), 428-436.

[5] Gerdts, M. (2008a). Global convergence of a nonsmooth Newton method for control-state constrained optimal control problems. SIAM Journal of Optimization, 19(1), 326-350.

[6] Gerdts, M. (2008b). A nonsmooth Newton's method for control-state constrained optimal control problems. Mathematics and Computers in Simulation, 79(4), 925-936.

[7] Gerdts, M. \& Kunkel, M. (2008). A nonsmooth Newton's method for discretized optimal control problems with state and control constraints. Journal of Industrial and Management Optimization, 4(2), 247-270.

[8] Goh, C. J. \& Teo, K. L. (1988). Control parametrization: a unified approach to optimal control problems with general constraints. Automatica, 24(1), 3-18.

[9] Halmos, P. R. (1974). Measure Theory. New York: Springer.

[10] Hartl, R. F., Sethi, S. P., \& Vickson, R. G. (1995). A survey of the maximum principles for optimal control problems with state constraints. SIAM Review, 37(2), 181-218.

[11] Jennings, L. S., Fisher, M. E., Teo, K. L., \& Goh, C. J. (2004). MISER3 optimal control software: Theory and user manual. Perth: The University of Western Australia.

[12] Lee, H. W. J., Teo, K. L., Rehbock, V., \& Jennings, L. S. (1999). Control parametrization enhancing technique for optimal discrete-valued control problems. Automatica, 35(8), 1401-1407.

[13] Loxton, R. C., Teo, K. L., \& Rehbock, V. (2008). Optimal control problems with multiple characteristic time points in the objective and constraints. Automatica, 44(11), 2923-2929.

[14] Martin, R. B. (1992). Optimal control drug scheduling of cancer chemotherapy. Automatica, 28(6), 1113-1123.

[15] Nocedal, J. and Wright, S. J. (1999). Numerical optimization. New York: Springer.

[16] Pytlak, R. \& Vinter, R. B. (1998). A feasible directions algorithm for optimal control problems with state and control constraints: Convergence analysis. SIAM Journal on Control and Optimization, 36(6), 1999-2019.

[17] Pytlak, R. \& Vinter, R. B. (1999). Feasible direction algorithm for optimal control problems with state and control constraints: Implementation. Journal of Optimization Theory and Applications, 101(3), 623-649.

[18] Rehbock, V. \& Livk, I. (2007). Optimal control of a batch crystallization process. Journal of Industrial and Management Optimization, 3(3), 585-596.

[19] Sakawa, Y. \& Shindo, Y. (1982). Optimal control of container cranes. Automatica, 18(3), 257-266.

[20] Schittkowski, K. (2007). NLPQLP: A fortran implementation of a sequential quadratic programming algorithm with distributed and non-monotone line search (version 2.24). Bayreuth: University of Bayreuth.

[21] Teo, K. L., Goh, C. J., \& Wong, K. H. (1991). A unified computational approach to optimal control problems. Essex: Longman Scientific \& Technical.
[22] Teo, K. L. \& Jennings, L. S. (1989). Nonlinear optimal control problems with continuous state inequality constraints. Journal of Optimization Theory and Applications, 63(1), 122.

[23] Teo, K. L., Jennings, L. S., Lee, H. W. J., \& Rehbock, V. (1998). The control parameterization enhancing transform for constrained optimal control problems. Journal of the Australian Mathematics Society - Series B, 40, 314-335.

[24] Teo, K. L., Rehbock, V., \& Jennings, L. S. (1993). A new computational algorithm for functional inequality constrained optimization problems. Automatica, 29(3), 789792 .

[25] Xu, X. \& Antsaklis, P. J. (2004). Optimal control of switched systems based on parameterization of the switching instants. IEEE Transactions on Automatic Control, 49(1), 2-16. 